\title{
Type I Kounis syndrome from Paclitaxel infusion
}

\author{
Benjamen Wang*, Anver Sethwala and Ronen Gurvitch \\ Department of Cardiology, Royal Melbourne Hospital, Melbourne, Victoria, Australia
}

\section{Introduction}

Coronary artery vasospasm leading to angina or myocardial infarction are among the most dangerous cardiotoxic effects of chemotherapeutic agents. The most well recognized association is with antimetabolite agents 5-fluorouracil and its prodrug capecitabine [1,2]. Paclitaxel is a microtubule-targeting drug of the Taxane family. It is widely used for the treatment of a range of cancers, namely breast, ovarian and lung. Its most commonly documented cardiac adverse effects have been bradycardia and heart block. Kounis syndrome has been described in a limited number of case reports explaining Paclitaxel and its association with acute coronary syndrome due to plaque rupture (Type 2 Kounis Syndrome) [3-5]. However, in these cases, patients have had pre-existing coronary artery disease or percutaneous coronary intervention. We report a patient treated for non-small cell lung cancer who presented with acute ST elevation shortly after a second paclitaxel infusion who was found to have no evidence of angiographic coronary artery disease. This is the first case report to our knowledge describing Type 1 Kounis Syndrome with Paclitaxel.

\section{Case report}

A 57-year-old gentleman with stage 3 lung adenocarcinoma was admitted to the oncology day unit for cycle 2 of paclitaxel and carboplatin. Other past medical history was that of current smoker, heavy alcohol consumption and hypertension. He had an uncomplicated first cycle of chemotherapy. He was premedicated with $8 \mathrm{mg}$ of dexamethasone. Shortly after commencing his second cycle with paclitaxel, before carboplatin was given, he developed acute onset dyspnoea, flushing and chest tightness. Clinical examination found him to be hypoxic at $82 \%$ on room air, tachypneic at $30 / \mathrm{min}$, hypotensive with a blood pressure of $85 / 50 \mathrm{mmHg}$ and tachycardic at 110 beats/min. Lung auscultation revealed diffuse wheeze. No cutaneous rash was evident. Electrocardiography (ECG) show inferior ST elevation with reciprocal changes in the precordial leads (Figure 1). Infusion of paclitaxel was ceased, salbutamol nebulizer, intravenous hydrocortisone, promethazine, and fluids were given along with $500 \mathrm{mcg}$ of intramuscular adrenaline and sublingual glyceryl trinitrate. The patient had a rapid improvement in symptoms and normalization of ECG. Bloods tests revealed a normal full blood count, renal function, liver function tests, creatinine kinase and only mildly elevated high sensitivity troponins of $26(\mathrm{~N}<16)$. Serum tryptase was normal. Semiurgent coronary angiography was performed which revealed only minor irregularities in the mid left anterior descending artery with an angiographically normal right coronary and left circumflex arteries.

Left ventriculography revealed normal left ventricular systolic function with no segmental wall motion abnormalities (Figure 2). A diagnosis of allergic coronary vasospasm of Kounis syndrome was made. The patient was treated with amlodipine $5 \mathrm{mg}$ daily with a strong recommendation not for further paclitaxel.

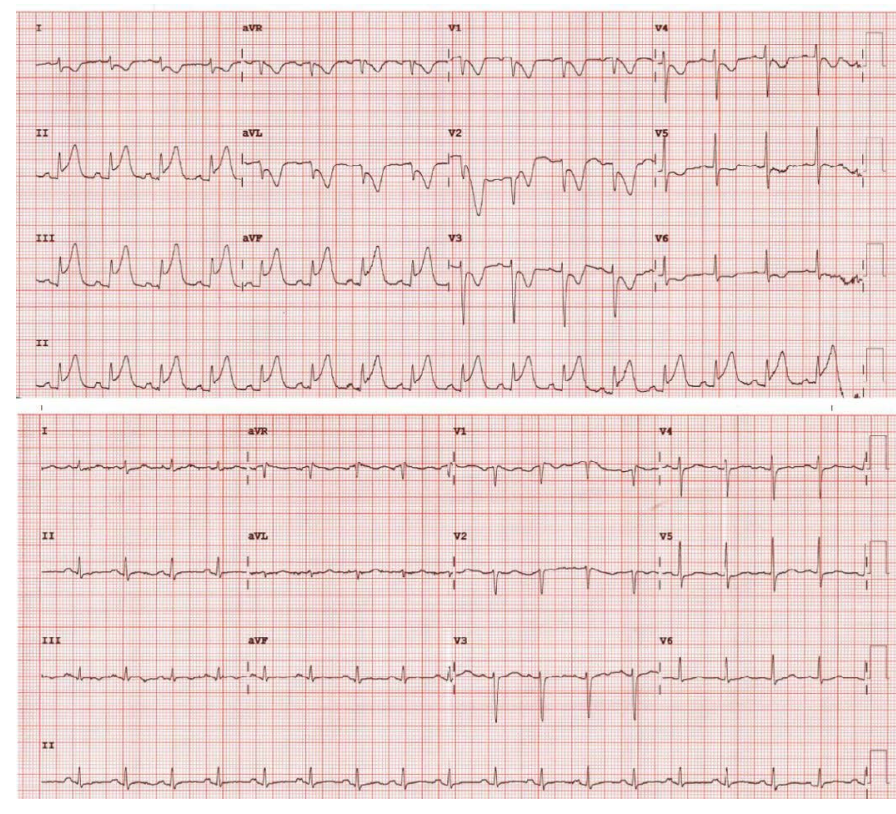

Figure 1. Top ECG showing ST elevation in inferior leads with reciprocal changes. Bottom: Post treatment ECG with normalized segments

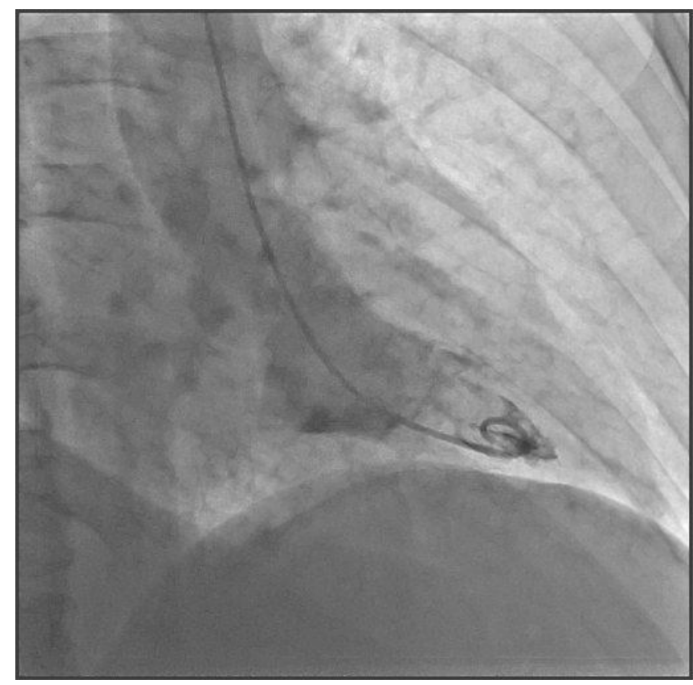

Figure 2. LV gram in end-systole showing normal systolic function

${ }^{\star}$ Correspondence to: Benjamen Wang, Department of Cardiology, Royal Melbourne Hospital, Melbourne, Victoria, Australia, E-mail: benjamen.m.wang@ gmail.com.

Received: March 18, 2020; Accepted: March 30, 2020; Published: April 08, 2020 


\section{Discussion and conclusion}

Kounis Syndrome is an acute coronary syndrome caused by an allergic reaction to drugs or other substances. It is differentiated from the classic non-allergic syndrome by the concurrent constellation of angioedema, wheezing, urticaria or hypotension etc. Elevated serum tryptase levels are diagnostic of anaphylaxis. However, a normal reading (as in our patient) does not exclude the diagnosis due to its low negative predictive value (54.3\%) [6]. The pathogenesis of Kounis Syndrome has been attributed to IgE mediated mast cell degranulation. The release of histamine, platelet activating factor, arachidonic acid and neutral proteases induce coronary spasm. These factors also contribute to a hypercoagulable state conducive to thromboses and acute ischemia [7]. Three variants of Kounis Syndrome have been proposed. Type I occurs in normal vessels and is likely the case in our patient. Type II manifests in atherosclerotic arteries where vasospasms erode or rupture pre-existing stable plaques, leading to a more typical picture of acute coronary syndrome. This variant has been more commonly described in case reports on patients receiving paclitaxel. Type III involves coronary thrombosis where evidence of eosinophils and mast cells in the thrombus is demonstrated on haematoxylineosin and Giemsa staining. There has only been one case report of suspected Type III Kounis Syndrome associated with paclitaxel use to our knowledge [8]. Although paclitaxel has been postulated to directly induce vasospasm through an increase in intracellular calcium concentrations [5], prompt resolution of symptoms, ECG changes and left ventricular function with anaphylaxis treatment strongly suggest a hypersensitivity mechanism instead. Cremophor EL (polyoxyethylated castor oil), in which paclitaxel is formulated and suspended, has also been a suspected allergenic agent [5, 9]. Higher incidences of severe hypersensitivity reactions to cremophor EL were involved in the early use of paclitaxel. Continuous cardiac monitoring was required [10]. This has been drastically mitigated by premedication protocols utilising intravenous steroids and antihistamines as was given to our patient. In managing patients with Type I Kounis Syndrome, we recommend switching to an alternate agent if possible. In our patient, paclitaxel has been ceased for carboplatin monotherapy with additional consideration for immunotherapy. In cases where the allergenic agent is needed, rechallenge should be undertaken with telemetry, premedication, GTN, non-dihydropyridine and calcium channel blockers given. An equivalent situation to our case with paclitaxel rechallenge has not been reported to our understanding. This case study highlights the risk of severe coronary spasm with paclitaxel even in patients with no previous ischemic heart disease or near normal coronary arteries.

\section{References}

1. Kounis NG, Koniari I, Patsouras N, Koutsogiannis N, Velissaris D, et al. (2017) Coronary vasospasm associated with 5-fluorouracil chemotherapy: Cardiac toxicity or cardiac hypersensitivity? Am J Emerg Med 35: 1769-1771. [Crossref]

2. Kounis NG, Tsigkas GG, Almpanis G, Mazarakis A (2012) Kounis syndrome is likely culprit of coronary vasospasm induced by capecitabine. J Oncol Pharm Pract 18: 316318. [Crossref]

3. Esber C, Breathett K, Sachak T, Moore S, Lilly S (2014) Acute Myocardial Infarction in Patient with Triple Negative Breast Cancer After Paclitaxel Infusion: A Case Report. Cardiol Res 5: 108-111. [Crossref]

4. Rawal GS, Yadav S, Kumar R (2016) Paclitaxel Induced Acute ST Elevation Myocardia Infarction: A Rare Case Report. J Clin Diagn Res 10: Xd01-xd02. [Crossref]

5. Nguyen-Ho P, Kleiman NS, Verani MS (2003) Acute myocardial infarction and cardiac arrest in a patient receiving paclitaxel. Can J Cardiol 19: 300-302. [Crossref]

6. Lin RY, Schwartz LB, Curry A, Pesola GR, Knight RJ, et al. (2000) Histamine and tryptase levels in patients with acute allergic reactions: An emergency departmentbased study. J Allergy Clin Immunol 106: 65-71. [Crossref]

7. Kounis NG, Mazarakis A, Tsigkas G, Giannopoulos S, Goudevenos J (2011) Kounis syndrome: a new twist on an old disease. Future Cardiol 7: 805-824. [Crossref]

8. Park SH, Byon JS, Lee SW, Lee SJ, Jin DK (2009) Coronary artery thrombosi associated with Paclitaxel in advanced ovarian cancer. Korean Circ J 39: 124-127. [Crossref]

9. Shah K, Gupta S, Ghosh J, Bajpai J, Maheshwari A (2012) Acute non-ST elevation myocardial infarction following paclitaxel administration for ovarian carcinoma: a case report and review of literature. J Cancer Res Ther 8: 442-444. [Crossref]

10. Arbuck SG, Strauss H, Rowinsky E, Christian M, Suffness M, et al. (1993) A reassessment of cardiac toxicity associated with Taxol. J Natl Cancer Inst Monogr 15: 117-130. [Crossref]

Copyright: (C2020 Wang B. This is an open-access article distributed under the terms of the Creative Commons Attribution License, which permits unrestricted use, distribution, and reproduction in any medium, provided the original author and source are credited. 